\title{
Deep Learning-based mobile robot for warehouse keeping
}

\author{
Akash Awasthi, A. Madhu Vamsi , P. Deeplakshmi
}

\begin{abstract}
As the warehouse plays a crucial role in the supply chain between the manufacturers and end-user or consumers, it is more important to adopt automation in large warehouses and industries. The large e-commerce companies like FLIPKART, AMAZON, SNAPDEAL etc. ship millions of goods and products from one place to another whose distance is in hundreds and even thousands of kilometres. These companies' warehouses sometimes are as big as nine football pitches or grounds which employ thousands of people for their inventory management. In this technically growing world, we need to reduce the manually done works by efficiently using the automation technology. This paper presents a modified design of Autonomous Inventory Management for Warehouses using automated mobile robots. This proposed work mainly focuses on robotic operations in logistics and warehouses, especially on obstacle avoidance and detection of the destination where it should halt (ie., location of desired object). It will adopt the surroundings by itself by using Machine Learning techniques. Once it reaches the destination, objects will be placed in tote by person and now it will reach preferred location in warehouse.
\end{abstract}

Keywords: Machine Learning, Deep Learning, Mobile Robot, Inventory Management.

\section{INTRODUCTION}

While we are in a technically growing world, there is surplus human work in warehouses in the management of goods from one place to another place that leads to huge employment. This problem can be solved by introduction of mobile platforms (mobile robots) that are automated for the purpose of doing all such good moving and management jobs [1]. The main problem for management of goods is, it requires the staffs to travel for long distances that result in many disadvantages like waste of time, waste of energy, etc [2]. We can employ Internet of Things (IOT) and IT infrastructure to come out of all kinds of problems. By introducing IOT and IT infrastructure, we can reduce the human work as well as time. The Warehouse applications mainly demand the flexibility development, safe, dependable solutions for efficient pick and place solutions [3]. The picking and placing of selected items from one place to other places are the basic operations in most of the robotic applications whether in large E-commerce

. Revised Manuscript Received on December 16, 2019

* Correspondence Author

Akash Awasthi*, Student, Dept. of CSE, Kalasalingam Academy of akashcseklu123@gmail.com

A. Madhu Vamsi, Student, Dept. of CSE, Kalasalingam Academy of Research and Education, Tamil Nadu, India. Email: madhumarvel143@gmail.com

P. Deeplakshmi, Professor, Dept. of CSE, Kalasalingam Academy of Research and Education, Tamil Nadu, India. Email: deepa.kumar@klu.ac.in Research and Education, Tamil Nadu, India. Email:

companies or in Warehouses. The current warehouse management system depends on the humans leads to huge employment so they can be replaced by deploying mobile robots [4].

The market also demands for flexible systems which allow the reduction of costs in the supply chain and increase competitiveness for the manufacturers by offering cost reduction to the consumers. The introduction of mobile robot for obstacle avoidance, picking and placing of desired objects from one place to another location without human intervention will make warehouse management much easier than the existing standard techniques. Once the robot is deployed, it will move along the desired path without hitting any object and it will search the desired object around it by using semantic segmentation techniques. Once it finds the desired object, it will stop there and it will wait for the user to place object in its tote and the preferred location where it should reach, it will take it as input from the user. It will reach the preferred location, where the object is delivered. Once the task is consummated successfully it will take the next desired object as input. Even though there are many astonishing techniques exists in warehouse management (like KIVA), small warehouses cannot procure them. This study mainly focuses on those small hold warehouses which cannot afford those high-level techniques.

\section{RELATED WORK}

The current existing obstacle avoidance robots are using conventional programmed robots. But these robots are not automated which cannot adopt their surroundings $[5,6]$.

The traditional standard technique involve manual effort in which human will manually do all the process by travelling nearly one football field area which is tedious work. There are few sophisticated techniques like [6] KIVA and GEEK robots which are incorporated in few warehouses but they are too costly (costs in the order of $\$ 1$ million to \$2 million) where a small warehouse holder cannot afford. Those warehouses who are willing to use these sophisticated robots, they have to redesign their warehouse to meet the requirements of the space and inventories should be placed in well-defined manner. For small scale warehouses, the authors in [13] created an RFID based warehouse robot which detects the object by scanning the RFID tag on the product. But in this RFID based robot, the path being followed by the robot is already pre-loaded into its PIC microcontroller which controls and navigates its movements.

\section{Published By:}


These systems are constrained with size and load they can deal with. These robots cannot adopt every environment. We have to convert the entire warehouse environment to use these robots, which can be too expensive. There are some other navigation modules for the wheel-based robots using fuzzy logic such as Goal Seeking Module, Static and Dynamic Obstacles avoidance Module. But these modules are too costly.

In our proposed work, the mobile manipulators can carry these racks autonomously to Human Pickers and then picked by workers standing in one place. The mobile platforms could be programmed to follow desired paths using the visual and magnetic markers [8,9]. Anyhow, it still needs some human workers to employ it. The picking operation involves the moving of items and places them in a tote $[10,11]$. The object that is picked maybe household items in larger which greatly varies in appearance, size, shape, hardness, and weight. We do not want to learn any preliminary stage of neural networks' weight. There are no constraints on how the items are organized and stored on the rack or tote [12].

\section{METHODOLOGY}

We are using ANN for controlling the velocity and obstacle avoidance of the autonomic vehicle under consideration. Our work is divided into two parts as hardware assembling and software development.

\section{A. Hardware Components}

We have used the following instruments to develop our project.

- Arduino Uno: It is the microcontroller that we have used to run the neural network code to control the vehicle. It takes input as the distance between the obstacle and the mobile bot. These distance values are given to neural network code to predict the velocity of the left and right wheel using the backpropagation algorithm implemented in Arduino using embedded C. Arduino is used to controp the LCD display which displays the velocity of left and right wheels.

- DC Motor: It is used to rotate the wheels of our mobile bot. We have used 5 Volt DC motor which is controlled by the motor driver and converts electrical energy to mechanical energy.

- Motor Driver: It is used to drive the motor. It converts low current signal to high current signal to drive the motor.

- Ultrasonic sensor: We have used the 3-pin ultrasonic sensor which gives the distance between the object and the mobile bot. It emits the ultrasonic wave and calculates the distance travelled to come back again.

- Wheels and chassis: We have used four wheels to move the mobile base and we have used chassis as a base to set all instruments.

- Battery: We have used 12 Volt battery to generate power to the motor and the Arduino.

- Potentiometer: We have used a potentiometer to control the intensity of the LCD and to control the mobile bot while training the neural network. We have used two Infrared sensors to calculate the speed of the left and right wheel velocity using black strips. The infrared sensor emits the IR waves and black color is a good absorbent so black strips are used to calculate one round. We have used 7446 IC as a voltage converter.

Kinect camera: We have used the Kinect camera to capture the image of the surroundings. These images are fed as an input to the ANN to recognize the destination.

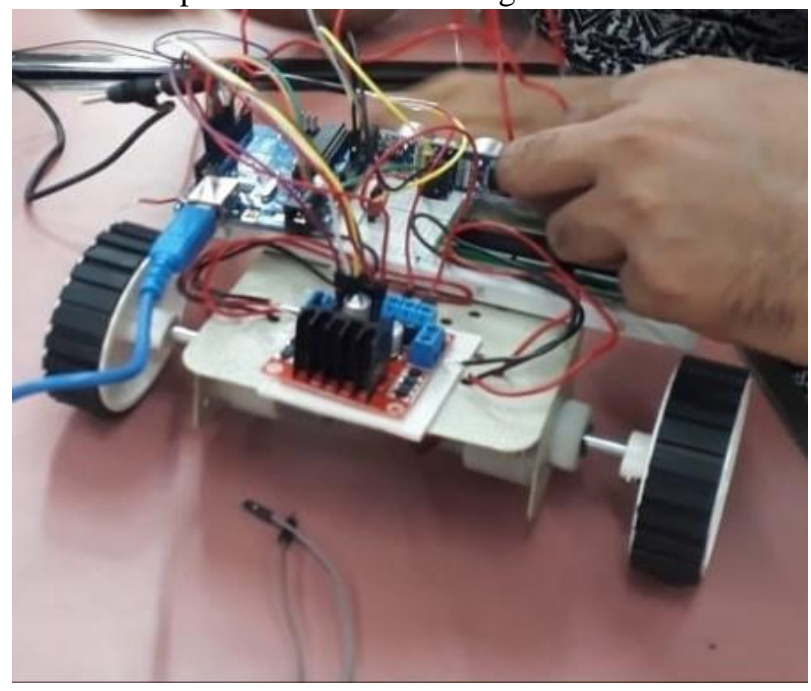

Fig. 1. Experimental setup

\section{B. Network Architecture}

We have used ANN for obstacle avoidance and object detection. We have used a three-layered neural network that is used for obstacle avoidance and to determine the destination. The proposed mobile manipulator can move in free space and carry the luggage from one location to another in the warehouses. We have used the backpropagation algorithm to train the network. Three-layered networks given in Fig. 2 for obstacle avoidance is explained as follows.

The network structure for obstacle avoidance: The three layers namely the input layer, the hidden layer, output layer are dense layers. The input layer has one neuron which takes the input $\mathrm{x}$, the ultrasonic sensor reading. The hidden layer consists of 10 neurons and the output layer consists of 2 neurons.

Parameters: Since the hidden layer has 10 neurons, there are 10 synaptic weights that connect from the input layer to the hidden layer. There are 20 synaptic weights that connect from the hidden layer to the output layer. There are 30 biases used in this network. We have initialized the value of these weights randomly. After training, we got the optimized weights and biases.

Hyperparameters: Hyperparameters are the learning rate and a number of neurons per layer. We have a grid search to optimize the hyperparameter.

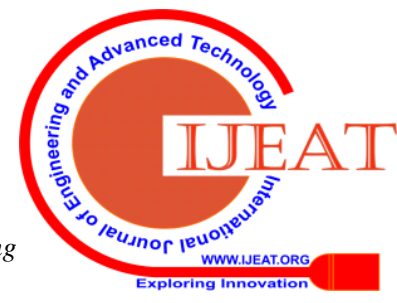




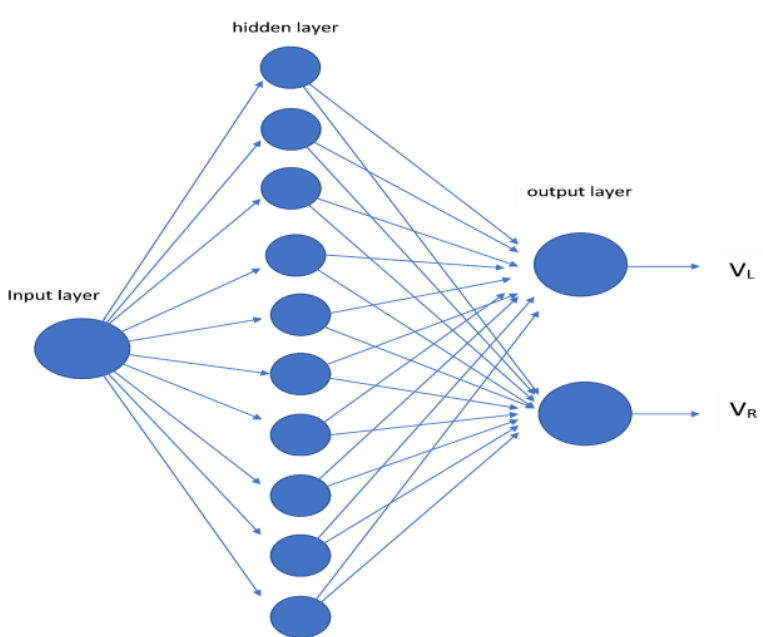

Fig. 2. Network Structure

Training: We have used the back-propagation algorithm to train our model. We have used gradient descent for weight optimization and we have used least square cost function to calculate the error. Weights have been updated after the mini-batches. Our model has taken 200 iterations to learn the optimal parameter. The equations used to calculate the new weight is as follows (2). It is a generalized delta rule.

Update Law

$$
\begin{array}{r}
\text { where } \quad w_{j k}(t+1)=w_{j k}(t)+\eta \delta_{j} x_{k} \\
\delta_{j}=V i(1-V i) \sum_{i}^{n} \delta j w_{j k}
\end{array}
$$

\section{TESTING}

We have tested our model for various ultrasonic sensor data. We have used a convolution neural network for image recognition.

\section{Image recognition and classification}

Deep learning plays an important role in developing autonomous systems. In the previous section, we have defined the obstacle avoidance algorithms for our autonomous vehicle using ANN. Destination detection is done by CNN. We have a Kinect camera at the center of the vehicle which captures the series of images. These images of the surroundings are sent as input to the CNN. CNN with dense layer and SoftMax node which outputs the probability distribution of an image frame captured by a kinetic camera. It is a multiclass image classification problem. We have trained our network with the destination images; the user selects the destination image on the screen of the robot. The class label corresponding to the destination image gets the highest probability among all classes. So, whenever it captures, it sends a signal to Arduino to stops the vehicle. We have used three convolution layers and one dense layer with a multi SoftMax node for probability corresponding to each class label. Our proposed CNN architecture is given in Fig.3.

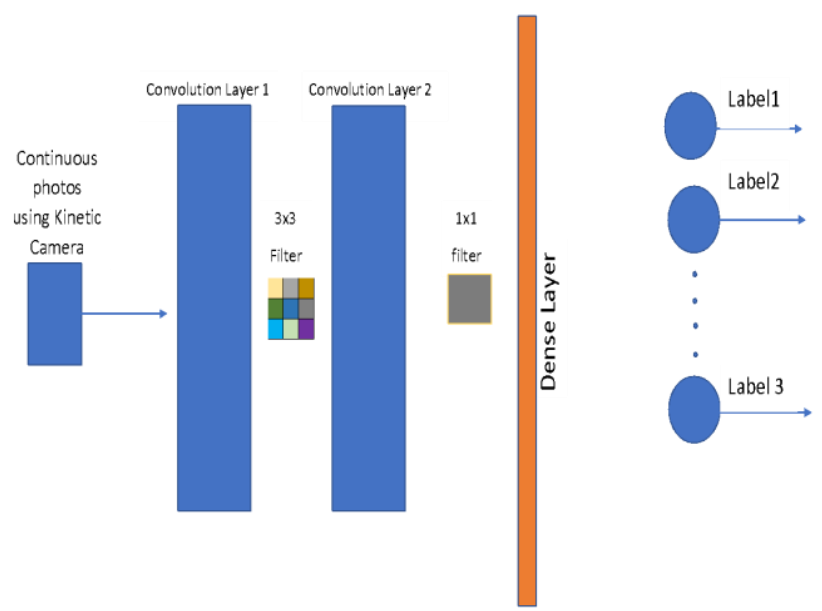

Fig. 3. Testing of the proposed method

\section{RESULTS}

We have used MATLAB for training and we have used the optimal weights and biases in embedded c code for operating the mobile bot. Our graph for root means square is given in Fig.4. First, we have trained our network with 1000 images and tested with 200 images. Our training error is 0.01 and the testing error is 0.2 . In graph, $\mathrm{X}$ axis represents the error and $\mathrm{Y}$-axis represents the number of epochs. We got an accuracy of $91 \%$.

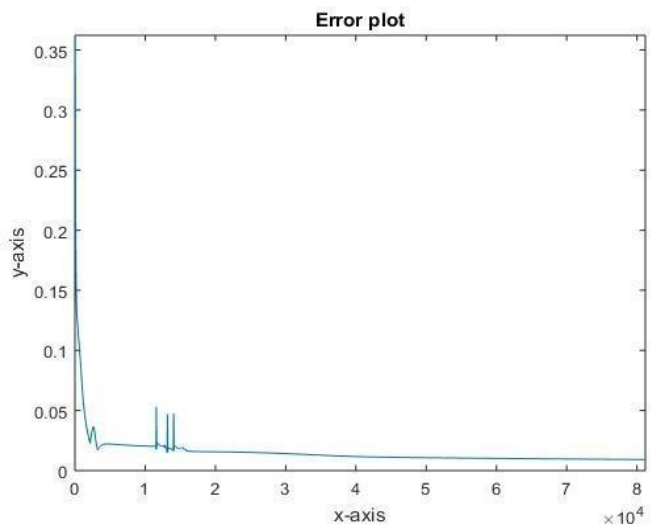

Fig. 4 Root Mean Square of proposed method

\section{CONCLUSION AND FUTURE WORK}

This mobile robot mainly focuses only on small hold warehouses that cannot afford a lot of money and whose warehouses are not well organized to use more sophisticated systems like KIVA and etc. We can add a manipulator to this mobile robot which will pick the desired detected objects using YOLO or Faster RCNN so that even picking process will be fully automated. In order to get exact contour of the object we can use pixel wise semantic segmentation technique like PSPnet,Mask RCNN instead of faster RCNN, but it would be more computationally expansive. 


\section{REFERENCES}

1. Jun-tao Li, Hong-Jian Liu. Design Optimization of Amazon Robotics. Automation, Control, and Intelligent Systems. Vol. 4, No. 2, 2016, pp. 48-52. DOI: 10.11648/j.acis.20160402.17

2. Kumar, Swagat, et al. "Design and Development of an automated Robotic Pick \& Stow System for an e-Commerce Warehouse." arXiv preprint arXiv:1703.02340 (2017). Kumar, Swagat, et al. "Design and Development of an automated Robotic Pick \& Stow System for an e-Commerce Warehouse." arXiv preprint arXiv:1703.02340 (2017).

3. Caron, F., G.Marchet, and.Perego. " Routing Policies and COI Based Storage Policies in Picker-to-Part Systems. International Journal of Production Research36 (3):713732.

4. Hamberg R, Verriet J. Automation in warehouse development. Springer; 2012 Jan 1.

5. Grosse. H.,C. H.Glock, M. Y.Jaber, and. P.Neumann.2015. "Incorporating Human Factors in Order Picking Planning Models: Framework and Research Opportunities. International Journal of Production Research53 (3):695717.

6. Grosse,E. H.,C. H.Glock, andW. P.Neumann.2017. "Human Factors in Order Picking: A Content Analysis of the Literature.International Journal of Production Research55 (5):12601276.

7. D'Andrea R. Guest editorial: A revolution in the warehouse: A retrospective on kiva systems and the grand challenges ahead. IEEE Transactions on Automation Science and Engineering. 2012 Oct;9(4):638-9.

8. Caron,F.,G.Marchet, and A.Perego.2000. "Optimal Layout in Low-Level Picker-to-Part Systems.International Journal of Production Research38 (1):101117.

9. Gagliardi,J. P.,J.Renaud, andA.Ruiz.2012. "Models for Automated Storage and Retrieval Systems: A Literature Review.International Journal of Production Research50 (24):71107125.

10. Lerher,T.2016. "Travel Time Model for Double-Deep Shuttle-Based Storage and Retrieval Systems.International Journal of Production Research54 (9):25192540.

11. Roodbergen,K. J., andR.De Koster.2001. "Routing Methods for Warehouses with Multiple Cross Aisles.International Journal of Production Research39 (9):18651883

12. De Koster,R.,E.Van der Poort, andM.Wolters.1999. "Efficient Orderbatching Methods in Warehouses.International Journal of Production Research37 (7):14791504.

13. Chuan, L. P., Johari, A., Wahab, M. H. A., Nor, D. M., Taujuddin, N. S. A. M., \& Ayob, M. E. (2007, November). An RFID warehouse robot. In 2007 International Conference on Intelligent and Advanced Systems (pp. 451-456). IEEE.

\section{AUTHORS PROFILE}

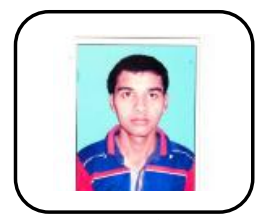

Akash Awasthi is a Computer Science undergraduate student in Kalasalingam Academy of research and Education. He had published 02 papers in field of high-performance computing and IoT. His major research area includes Machine Learning, Computer Vision, High-performance computing, Deep Learning, IoT and Artificial Intelligence.

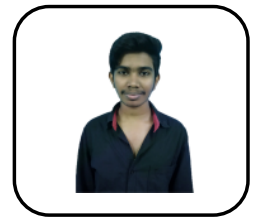

A. Madhu Vamsi is a Computer Science undergraduate student in Kalasalingam Academy of research and Education. He had published 02 papers in field of high-performance computing and IoT. His major research area includes Machine Learning, Deep Learning and Artificial Intelligence.

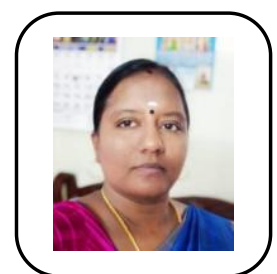

Dr. P. Deepalakshmi is currently working as a Professor in Department of Computer Science and Engineering at Kalasalingam Academy of Research and Education (KARE), Virudhunagar, Tamilnadu, India. She is also serving as Dean, School of Computing. Her research interest includes Optimization Techniques, Network
Routing, Distributed Computing, Network Security, Data Analytics, Machine Learning Techniques. She also takes care of KARE ACM student chapter as faculty mentor. Contact her at deepa.kumar@klu.ac.in 